\title{
Chain-mixing behavior at interface between polystyrene brushes and polystyrene matrices
}

\author{
Hiroshi Arita ${ }^{1}$, Koji Mitamura ${ }^{2}$, Motoyasu Kobayashi ${ }^{2}$, Norifumi L Yamada ${ }^{3}$, Hiroshi Jinnai ${ }^{2,4,5}$ and \\ Atsushi Takahara ${ }^{2,4,5}$
}

The effect of the molecular weight dispersity (MWD) on the interfacial width between an end-tethered polymer brush and a free polymer matrix has been experimentally investigated by neutron reflectivity (NR) measurements. We prepared high-density hydrogenous polystyrene (hPS) brushes with different MWDs by surface-initiated controlled radical polymerization. Unbound (free) deuterated polystyrene (dPS) with a similar molecular weight to the hPS was mounted on the hPS brush to form bilayer films and annealed at $398 \mathrm{~K}$ for $2-120 \mathrm{~min}$. The interfacial width between the narrow-MWD hPS and dPS matrix was relatively unchanged after thermal annealing because of the high graft density and similar molecular architectures (dry-brush regime). However, the broad-MWD hPS brush showed a large interfacial width (wet-brush regime) due to the extension of the longer chains into the dPS matrix and intermixing. The interface width increased rapidly from 2 to $20 \mathrm{~nm}$ after annealing for 5 min and increased slowly to $30 \mathrm{~nm}$ after $120 \mathrm{~min}$. The volume fraction profile of the broad-MWD hPS brush showed a characteristic gradient distribution as the distance from the substrate surface increased, consistent with the theoretical simulations by Laub and Koberstein. ${ }^{7}$ Polydisperse brushes are expected to improve the adhesion and interfacial toughness at solid-polymer interfaces.

Polymer Journal (2013) 45, 117-123; doi:10.1038/pj.2012.198; published online 21 November 2012

Keywords: chain-mixing behavior; polystyrene; polymer brush; NR; interface

\section{INTRODUCTION}

Polymer brushes, assemblies of surface-tethered polymers on solid substrates, are useful for many physicochemical applications such as wetting, adhesion and colloid stabilization. In many composite and multiphase materials, polymer brushes have an important role in improving the affinity of the substrate surface to a polymer matrix; those at the interface of a polymer matrix and non-polymeric substrate usually increase the bonding strength of the two materials. The interfacial strength depends on the extent to which the brush polymer penetrates the bulk and on the entanglement of the brush and bulk polymers. However, in some cases, the interfacial strength is not increased even for chemically identical polymer brushes and matrices because the intermixing of the brush and bulk polymer is dominated by the chain length and graft density $(\sigma)$ of the brushes. ${ }^{1}$ The graft density is the average number of grafted chains per unit surface area $a^{2}$ of the solid surface, where $a$ is the monomer size. In the case of nanoparticle suspensions in a polymer matrix, for example, polymer grafting enhances the particle dispersion when the grafting density is low because repulsion of the grafted layers prevents van der Waals attraction between the particles. However, if $\sigma$ is too high, then flocculation of the nanoparticles takes place because of the effective entropic attraction between the grafted layers. ${ }^{2}$

Intermixing between a polymer brush and the unbound polymer chains in a matrix is an interesting problem typically separated into 'wet' (swollen) brush and 'dry' (unswollen) brush regimes. ${ }^{3}$ The degree of interpenetration of a brush in contact with unbound polymers with a chemically similar structure is described by a phase diagram depending on $N, P$, and $\sigma$, where $N$ and $P$ are the degree of polymerization $\left(D_{\mathrm{p}}\right)$ of the polymer brush and bulk (unbound) polymer, respectively., ${ }^{4,5}$

When the grafting density is sufficiently small $\left(\sigma<N^{-1}\right)$, there is little contact between neighboring brush chains, which behave as isolated coils to form a mushroom-like structure. In this regime, the height of the brush is of the order of the Flory radius of the coil. For values of $\sigma>N^{-1}$ and $<N^{-1 / 2}$, neighboring brush chains begin to come into contact and therefore stretch away from the surface. In addition, the free polymer chains in the matrix can penetrate into the brush layer and induce brush swelling, resulting in the wet-brush regime.

When $\sigma$ is much higher than $N^{-1 / 2}$, the dry-brush regime appears. The polymer brush forms a more extended chain structure owing to the excluded volume effect caused by the monomer-monomer

${ }^{1}$ Department of Chemistry and Biochemistry, Graduate School of Engineering, Kyushu University, Fukuoka, Japan; ${ }^{2} J a p a n$ Science and Technology Agency, ERATO Takahara Soft Interfaces Project, Fukuoka, Japan; ${ }^{3}$ Institute of Material Structure Science, High Energy Accelerator Research Organization (KEK), Tokai, Japan; ${ }^{4}$ Institute for Materials Chemistry and Engineering, Kyushu University, Fukuoka, Japan and 5 International Institute for Carbon-Neutral Energy Research (WPI-I2CNER), Kyushu University, Fukuoka, Japan Correspondence: Professor A Takahara, Institute for Materials Chemistry and Engineering, Kyushu University, 744 Motooka, Nishi-ku, Fukuoka 819-0395, Japan. E-mail: takahara@cstf.kyushu-u.ac.jp

Received 27 August 2012; revised 5 October 2012; accepted 5 October 2012; published online 21 November 2012 
interactions between the grafted chains. In this regime, the free polymer cannot penetrate into the brush layer because intermixing is entropically unfavorable; the entropic gain associated with the mixing of free polymer chains and the brush does not compensate the elastic deformation of the brush chains. The transition from the wet- to drybrush regime depends on $N$ and $P$, as well as on $\sigma$. The boundary conditions for the regime transition are described as follows.

$$
\begin{gathered}
\sigma>\frac{1}{\sqrt{P}} \quad(P<N) \\
\sigma>\frac{1}{\sqrt{N}} \quad(P<N)
\end{gathered}
$$

The interface between a brush layer and bulk polymer with chemically identical chains has been studied theoretically on the basis of selfconsistent field theory and experimentally by examining the autophobic wetting behavior of polymer chains. However, the effect of the molecular weight dispersity (MWD) on the interfacial thickness between the brush and bulk polymer has been largely ignored in previous studies.

The molecular weights of synthetic polymers have distribution even if they were prepared by controlled or living polymerization. Polymer brushes prepared by a 'grafting from' method will contain polymer chains of various lengths. Therefore, the number of grafting polymers within a certain area on the outermost surface must be lower than the anchoring density at the grafting surface. Intermixing of the brush and bulk polymers is expected at the interface even in the dry-brush state. The behavior of a brush containing polymers of two different molecular weights was demonstrated theoretically by Milner et al. ${ }^{6}$ who used self-consistent field theory to reveal that the concentration profile of the bimodal brush at the interface changed drastically from that of a monodispersed brush. Laub and Koberstein ${ }^{7}$ further developed the systematic simulation for a polymer brush with an arbitrary MWD, graft density, and $D_{\mathrm{p}}$ to investigate the width of the interface between the brush and polymer matrix. They showed clearly that the interfacial mixing of the brush and matrix polymer increased as the MWD increased. Polymer brushes with a broad MWD increase the possibility of entanglement of the brush chains and free polymer chains in the matrix, which is also expected to improve the interfacial toughness and adhesive strength between the brush substrate and polymer matrix. However, the dependence of the interfacial structure on polydispersity is yet to be experimentally investigated.

In this study, the intermixing behavior at the interface between a hydrogenous polystyrene (hPS) brush and a free deuterated polystyrene (dPS) was investigated by neutron reflectivity (NR) measurements. We prepared three hPS brushes with different graft density and MWDs by surface-initiated atom transfer radical polymerization (SI-ATRP). Both brushes are theoretically dry brushes having a high $\sigma$ and similar degree of polymerization $N$ to the corresponding $P$ of the dPS. The Flory-Huggins interaction parameter $\chi$ between the hPS and dPS is negligibly small, and therefore, interpenetration is expected to proceed in an athermal condition dominated by entropic favor. We focus on the effect of MWD on the intermixing of the free dPS into the brush layer by thermal annealing at a temperature above the glass transition temperature $\left(T_{\mathrm{g}}\right)$. The time evolution of the interpenetration thickness during the annealing is also discussed.

\section{EXPERIMENTAL PROCEDURE}

\section{Materials}

Copper(I) bromide (CuBr, Wako Pure Chemical Industries, Ltd., Osaka, Japan, 98\%) was washed successively with acetic acid and ethanol, and then dried under vacuum. Ethyl 2-bromoisobutyrate (EB, Tokyo Chemical Industry Co., Ltd., Tokyo, Japan, 99\%) was distilled from calcium hydride before use, and tris-(2-(dimethyl)aminoethyl)amine $\left(\mathrm{Me}_{6} \mathrm{TREN}\right)$ was synthesized using previously reported procedures. ${ }^{8}$ Pentamethyldiethylenetriamine (PMDETA, Aldrich, St Louis, MO, USA) was used as received. A styrene monomer (Wako Pure Chemical Industries, Ltd., 99.0\%) was washed with $\mathrm{NaOH}$ aq. to remove the stabilizer, dried over magnesium sulfate and then purified by distillation under reduced pressure in the presence of calcium hydride. The dPS (number-average molecular weight $\left(M_{\mathrm{n}}\right)=38500$; MWD index $\left(M_{\mathrm{w}} / M_{\mathrm{n}}\right)=$ 1.07) was purchased from Polymer Source, Inc. (Dorval, Quebec, Canada). Water for floating the self-standing dPS film was purified using the Simpli Lab system (Millipore Co., Ltd., Billerica, MA, USA). The synthesis procedure for the surface initiator 11-(2'-bromo-2'-methyl)propionyloxyundecenyltrimethoxysilane (BMS) and 11-(2'-methyl)propionyloxyundecenyltrimethoxysilane are described in the Supplementary Information.

\section{Silicon wafers immobilized with surface initiator}

Silicon wafers $\left(40 \times 15 \times 0.5 \mathrm{~mm}^{3}\right)$ were washed with piranha solution at $373 \mathrm{~K}$ for $1 \mathrm{~h}$ and then exposed to vacuum-ultraviolet rays (wavelength $\lambda=172$ $\mathrm{nm})$ for $10 \mathrm{~min}$ under reduced pressure $(30 \mathrm{~Pa})$. (Caution: piranha solution, consisting of concentrated sulfuric acid and hydrogen peroxide, is a dangerous solution that should be handled with care.) The hydrophilic silicon wafers were immersed in $0.5 \%$ BMS/dry toluene solution at room temperature under nitrogen gas in a glove box for $4 \mathrm{~h}$. The wafers were rinsed with ethanol (HPLC grade, Wako Chemical Co.) and annealed at $383 \mathrm{~K}$ for $3 \mathrm{~h}$ to obtain BMSimmobilized silicon. The graft density of the surface initiation site on the silicon wafer was tuned by the BMS/11-(2'-methyl)propionyloxyundecenyltrimethoxysilane mixing ratio $(2 / 8-1 / 50, v / v)$ in dry toluene solution. Silicon wafers immersed in the mixed BMS-HMS toluene solution yielded hPS brushes with a low graft density by SI-ATRP of the styrene.

\section{Preparation of hPS brushes}

SI-ATRP of the styrene was carried out at $348 \mathrm{~K}$ using a $\mathrm{CuBr} / \mathrm{Me}_{6} \mathrm{TREN}$ catalyst in the presence of free initiator EB under argon to simultaneously generate a PS brush from the substrate and free (unbound) PS from the EB. The reaction conditions are described in detail in the Supplementary Information. The resulting hPS had a predictable molecular weight $\left(M_{\mathrm{n}}=\right.$ $38700)$ and relatively narrow MWD $\left(M_{\mathrm{w}} / M_{\mathrm{n}}=1.12\right)$. An hPS brush with a broad MWD $\left(M_{\mathrm{n}}=51900\right.$ and $\left.M_{\mathrm{w}} / M_{\mathrm{n}}=2.02\right)$ was also prepared by SI-ATRP by using $\mathrm{CuBr}$ and PMDETA in anisole at $383 \mathrm{~K}$. The hPS brush-immobilized silicon wafers were washed with toluene using a Soxhlet apparatus for $6 \mathrm{~h}$ to remove the free polymer adsorbed on the surface and were dried under reduced pressure.

\section{Preparation of dPS bilayer film on hPS brush}

The dPS was dissolved in toluene and filtered by a chromatodisk with $0.45-\mu \mathrm{m}$ pores to prepare $2.8 \mathrm{wt} \%$ dPS toluene solution. A $0.5-\mathrm{ml}$ volume of the solution was dropped on a glass substrate and spin-coated at 3000 r.p.m. for $20 \mathrm{~s}$ and then air dried. Subsequently, the resulting dPS thin film was floated off in water ${ }^{9,10}$ and picked up with the hPS brush substrate from above. The $\mathrm{dPS}$ film/hPS brush bilayer film was dried at room temperature under vacuum for $2 \mathrm{~h}$.

\section{Characterization}

Size exclusion chromatography was performed at $313 \mathrm{~K}$ on a Tosoh HLC-8220 GPC system (Tokyo, Japan) connected to three TSKgel SuperH PS gel columns (6000, 4000 and 2500; Tosoh, Yamaguchi, Japan) and equipped with a refractive index and ultraviolet-visible detector. Tetrahydrofuran was used as the eluent at a flow rate of $0.6 \mathrm{ml} \mathrm{min}^{-1}$. Polystyrene standards $\left(M_{\mathrm{n}}=1060\right.$ $\left.1090000 ; M_{\mathrm{w}} / M_{\mathrm{n}}=1.02-1.08\right)$ were used for the calibration to estimate the $M_{\mathrm{n}}$ and MWD values of the polymers.

A spectroscopic ellipsometer (MASS-102, Five Lab Co., Ltd., Kanagawa, Japan) with a xenon arc lamp $(\lambda=380-890 \mathrm{~nm})$ was used to determine the thickness of the brush thin film. The fixed incident angle was $70^{\circ}$, and the refractive index was assumed to be 1.59 . 
Atomic force microscope observations were carried out in air at room temperature using an SPA 400 with an SPI 4000 controller (SII Nano Technology Inc., Chiba, Japan) and a SiN integrated tip on a commercial rectangular cantilever (OMCL-RC800PB-1 (Au coated), Olympus Co., Ltd., Tokyo, Japan) with a spring constant of $0.11 \mathrm{~N} \mathrm{~m}^{-1}$.

NR measurements were carried out using a soft interface analyzer time-offlight-type reflectometer ${ }^{1-13}$ installed in BL-16 at Materials and Life Science Facility in Japan Proton Accelerator Research Complex (Tokai, Japan). Japan Proton Accelerator Research Complex provided 25-Hz-pulsed neutron radiation at $210 \mathrm{~kW}$. The wavelength of the incident neutrons was tuned to around $0.20-0.88 \mathrm{~nm}$ by a disk chopper. The neutron beam irradiated the interface between air and polymer on silicon substrate to the silicon substrate, and the reflected neutrons were collected by a $2 \mathrm{D}$ scintillation detector. The neutron momentum transfer vector is $q=(4 \pi / \lambda) \sin \theta$, where $\theta$ is the angle of specular reflection. A $30-\mathrm{mm}$ beam footprint was maintained on the sample surface by using incident slits. The MOTOFIT program ${ }^{14}$ was used to fit the reflectivity profiles to modeled scattering length density (SLD) layers, in which the thickness of each layer, SLD and Gaussian roughness were optimized to minimize the least-squares of the measured and calculated reflectivity curves. The SLD of the dPS, hPS, $\mathrm{SiO}_{2}$ and $\mathrm{Si}$ used in this study were $6.46 \times 10^{-4}$, $1.43 \times 10^{-4}, 3.47 \times 10^{-4}$ and $2.07 \times 10^{-4} \mathrm{~nm}^{-2}$, respectively. ${ }^{15,16}$

The bilayer films were annealed under reduced pressure for the intermixing experiment. The samples were quickly transferred to a thermal stage cell preheated to $398 \mathrm{~K}$ and equipped with a vacuum cock, covered with an aluminum plate and then evacuated immediately. The annealing time initiated from this point. After annealing for a given time $(2-120 \mathrm{~min}), \mathrm{N}_{2}$ gas was introduced into the thermal stage cell, and the sample was rapidly picked up and quenched on a stainless steel plate at $298 \mathrm{~K}$. Each NR measurement was conducted at $298 \mathrm{~K}$.

\section{RESULTS AND DISCUSSION}

\section{Intermixing behavior of narrow-MWD hPS brushes}

Three types of hPS brushes with different graft densities and MWDs were prepared by the SI-ATRP of styrene from silicon wafers with various alkyl-bromide initiator adsorption ratios. The molecular weight and MWD of the hPS brushes, estimated from an size exclusion chromatography analysis of the free hPS simultaneously obtained during the SI-ATRP, are listed in Table 1. The hPS-1 and hPS-2 brushes have relatively narrow MWDs $\left(M_{\mathrm{w}} / M_{\mathrm{n}}=1.12\right)$ but different graft densities, whereas the hPS-1 and hPS-3 brushes have a similar $\sigma$ value $(0.317-0.350)$ but different MWDs. The $\sigma$ values were calculated using the $M_{\mathrm{n}}$ of the hPS brush, the bulk polystyrene density $\rho\left(1.05 \mathrm{~g} \mathrm{ml}^{-1}\right)$, the thickness of the brush $L_{\mathrm{d}}(\mathrm{nm})$, Avogadro's number $N_{\mathrm{A}}$ and the cross-sectional area of the polystyrene $A$ $\left(0.442 \mathrm{~nm}^{2}\right)^{17}$ as

$$
\sigma=\frac{\rho L_{d} N_{\mathrm{A}} A}{M_{n}} \times 10^{-21}
$$

The graft density is typically the number of chains per square nanometer; however, the $\sigma$ values calculated in this study are dimensionless owing to scaling by the cross-sectional area $A$. In other words, $\sigma$ is the polymer coverage on the substrate surface.

Table 1 Characteristics of polystyrenes used in this study

\begin{tabular}{lccccc}
\hline Sample & $M_{\mathrm{n}}$ & $D_{p}{ }^{\mathrm{a}}$ & $M_{\mathrm{w}} / M_{\mathrm{n}}$ & Thickness $^{\mathrm{b}}$ & $\sigma^{\mathrm{c}}$ \\
\hline hPS-1 & 38700 & $N=372$ & 1.12 & 44 & 0.317 \\
hPS-2 & 38700 & $N=372$ & 1.12 & 6 & 0.0414 \\
hPS-3 & 51900 & $N=499$ & 2.02 & 65 & 0.350 \\
dPS & 38500 & $P=344$ & 1.07 & - & -
\end{tabular}

Abbreviations: dPS, deuterated polystyrene; hPS, hydrogenous polystyrene.

aDegree of polymerization.
bThickness in air-dried state was estimated by ellipsometer.

'Graft density was calculated by equation (3).
The phase diagram in Figure 1 shows the dry and wet regimes according to the scaling laws of a hPS brush with a $D_{\mathrm{p}}$ of $N$ in contact with the dPS film with $P=344$. When $N>P$, the boundary between the dry- and wet-brush regimes is determined theoretically to be $\sigma=P^{-1 / 2}=0.054$. Accordingly, the bilayers of the dPS/hPS- 1 and $\mathrm{dPS} / \mathrm{hPS}-3$ brushes are in the dry-brush region, whereas that of the $\mathrm{dPS} / \mathrm{hPS}-2$ brush is located in the wet-brush region.

Figure 2 shows the reflectivity profile of the dPS/hPS- 1 brush bilayer on the silicon substrate at an angular resolution $\Delta \theta / \theta$ of $3.0 \%$. The solid line is the fit on the basis of SLD calculations assuming a four-layer model of $\mathrm{dPS} / \mathrm{hPS} / \mathrm{SiO}_{2} / \mathrm{Si}$ (Figure $2 \mathrm{~b}$ ). Well-defined fringes were observed in a $q$ range of $0.15-0.6 \mathrm{~nm}^{-1}$. The fringe spacing in the NR profile is predominantly governed by the thickness of the dPS layer owing to the large difference in the SLD between air and the APS compared with that between the hPS. Fits to the NR curves gave dPS and hPS brush thicknesses of 110 and $44 \mathrm{~nm}$, respectively. On the basis of the SLD profile in Figure 2b), the hPS volume fraction $\phi(z)$ as a function of depth $z$ from the center of the interface was calculated as

$$
\phi(z)=\left(\mathrm{SLD}_{\mathrm{dPS}}-\mathrm{SLD}(\mathrm{z})\right) /\left(\mathrm{SLD}_{\mathrm{dPS}}-\mathrm{SLD}_{\mathrm{hPS}}\right)
$$

The center of the interface was defined as $z=0$ when the hPS volume fraction was 0.5 . Figure 5 a shows the $\phi(z)$ profile at the dPS/ hPS-1 brush interface before and after annealing. We approximated the $\phi(z)$ profile using two error functions, ${ }^{18}$

$$
\begin{aligned}
& \phi(z)=\frac{1}{2}-\frac{1}{2} \operatorname{erf}\left(\frac{-z}{\sigma_{s} \sqrt{2}}\right) \quad(z>0) \\
& \phi(z)=\frac{1}{2}+\frac{1}{2} \operatorname{erf}\left(\frac{z}{\sigma_{s} \sqrt{2}}\right) \quad(z<0)
\end{aligned}
$$

where $\sigma_{\mathrm{s}}$ is the s.d., and the size of the interfacial width is determined as $2 \sigma_{\mathrm{s}}$. The interface between the hPS brush and bulk dPS in the unannealed (as-prepared) sample was relatively sharp $\left(2 \sigma_{\mathrm{s}}=2.5 \mathrm{~nm}\right)$.

In this work, wet- and dry-brush behavior will be discussed on the basis of the degree of intermixing of the brush and free polymer, which was estimated by the size of the interfacial width. However, it is not easy to determine the interfacial width at the asymmetric

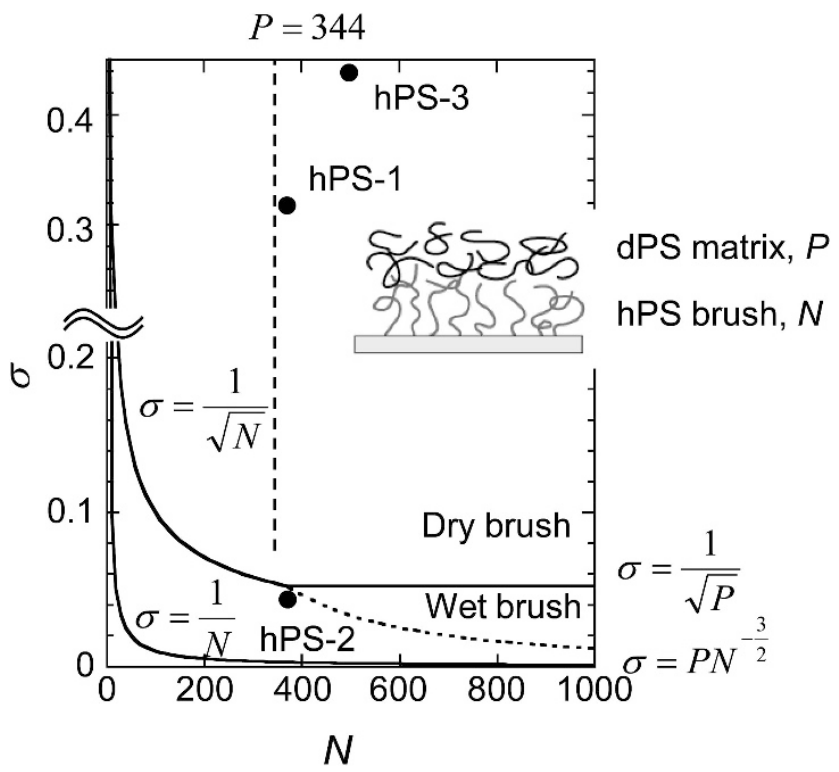

Figure 1 Phase diagram of polymer brush state, and schematic image of a bilayer of hydrogenous polystyrene (hPS) brush and free deuterated polystyrene (dPS) film. 
geometry of free polymer and the brush. In addition, three hPS brushes have different MWDs, graft density and interfacial mixing structure. It is difficult to find the universal functions to describe the interfacial structures. Therefore, we used the s.d. of the error functions in order to compare the degree of intermixing of the three types of hPS brushes/free dPS films, although the symmetric error functions cannot describe the interfacial fraction.

The hPS brush and free dPS intermixing was investigated by an 'anneal-quench' procedure in which the sample was heated for a given time above $T_{\mathrm{g}}$ and then rapidly quenched to room temperature, after which a full NR profile was collected. This procedure was repeated for each successive annealing time. As the annealing time at $398 \mathrm{~K}$ lengthens (Figure $2 \mathrm{a}$ ), the fringes at higher $q$ values become unclear because of the intermixing. The interfacial thickness of the dPS/hPS-1 brush increased from 2 to $10 \mathrm{~nm}$ by annealing for $120 \mathrm{~min}$, but the interfacial thickness increased quickly after $2 \mathrm{~min}$ and saturates at $10 \mathrm{~nm}$. The SLD profiles and the volume fraction of the hPS brushes after annealing for different durations overlap for $-10<z<10 \mathrm{~nm}$
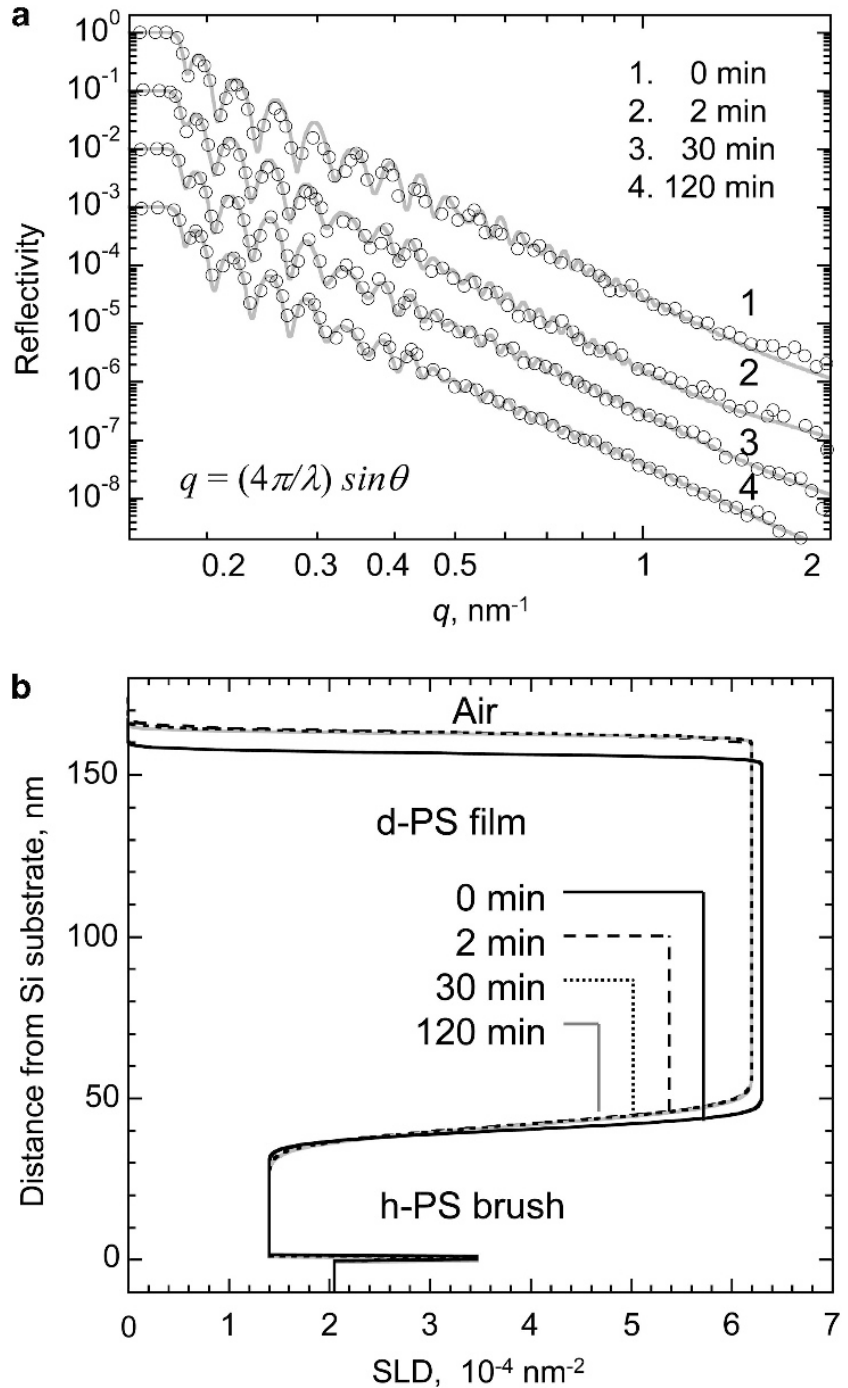

Figure 2 (a) Neutron reflectivity curves (open circles) of hydrogenous polystyrene (hPS)-1 brush/deuterated polystyrene (dPS) bilayer film annealed at $398 \mathrm{~K}$ for $0,2,30$ and $120 \mathrm{~min}$, and the corresponding fit (gray line) calculated on the basis of (b) the scattering length density profiles along with distance from the silicon surface.
(Figure 5a), indicating that the dPS could not penetrate the brush layer owing to the high graft density.

The thickness of the hPS-1 brush/dPS bilayer increased by ca $5 \mathrm{~nm}$, as shown in Figure 2b. As mention in the Experimental procedure section, free dPS film was prepared on Si wafer by spin coat method without thermal annealing, and transferred on hPS brush surface. The spin-coated dPS would form a relatively collapsed chain structure. We suppose that the dPS chains might be relaxed and recovered by the entropic elasticity during the thermal annealing process to increase the thickness of dPS layer.

For the hPS-2 brush, shown in Figure 3, the wet-brush regime is expected to appear owing to the low graft density. The initial thickness of the hPS-2 brush was $6 \mathrm{~nm}$, but the brush began to stretch toward the matrix after annealing at $398 \mathrm{~K}$. The interfacial width $\left(2 \sigma_{\mathrm{s}}\right)$ increased from 1.8 to $12 \mathrm{~nm}$ after annealing for $120 \mathrm{~min}$. The SLD near the $\mathrm{SiO}_{2}$ surface after annealing was $3.4 \times 10^{-4} \mathrm{~nm}^{-2}$, which is much higher than that of the hPS $\left(1.43 \times 10^{-4} \mathrm{~nm}^{-2}\right)$, indicating that the free dPS penetrated through the brush layer and
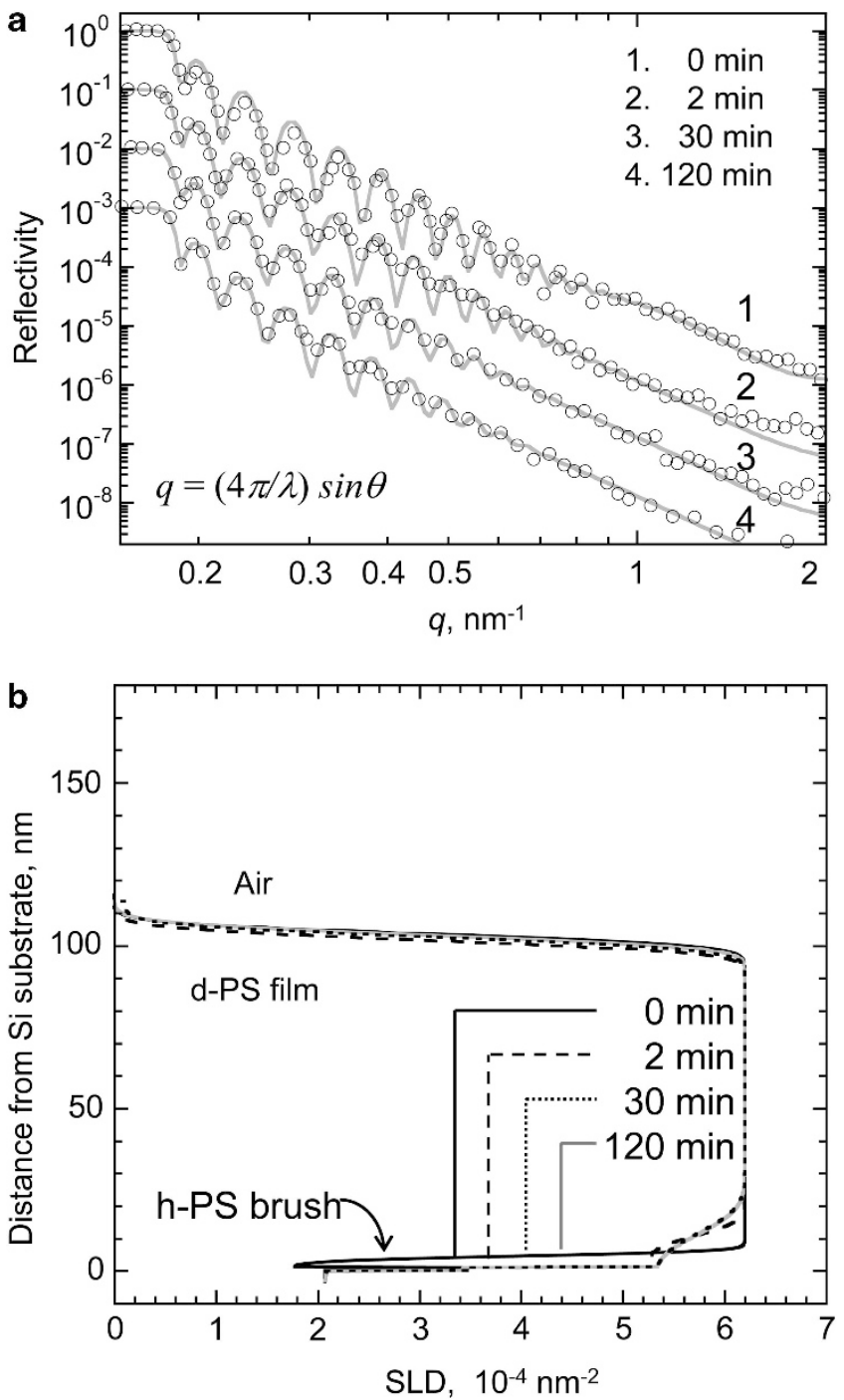

Figure 3 (a) Neutron reflectivity curves (open circles) of hydrogenous polystyrene (hPS)-2 brush/deuterated polystyrene (dPS) bilayer film annealed at $398 \mathrm{~K}$ for $0,2,30$, and $120 \mathrm{~min}$, and the corresponding fit (gray line) calculated on the basis of (b) the scattering length density profiles along with distance from the silicon surface. 
reached the grafting surface. Simultaneously, the hPS brush chains were forced to stretch toward the dPS matrix leading to the increase in the interfacial width. The height of the swollen hPS brush increased to $20 \mathrm{~nm}$. We have thus experimentally confirmed the theoretically predicted wet-brush behavior of the hPS-2 brush.

More precisely, the height (thickness) of the swollen brush $(H)$ is determined by two physical contributions: (a) the entropy of the mixing between the $N$-brush chains and $P$-polymer chains, and (b) the elastic energy dominated by the conformation of the brush chains under stretching and compression. ${ }^{3}$ The height of a semi-diluted brush can be approximated as ${ }^{5}$

$$
H=N \sigma^{\frac{1}{3}} P^{-\frac{1}{3}}
$$

Using the $N$ and $\sigma$ values of the hPS-2 brush and the $P$-value of the dPS, $H$ is estimated to be $18 \mathrm{~nm}$, which is the same order of magnitude as that observed in the NR measurements.
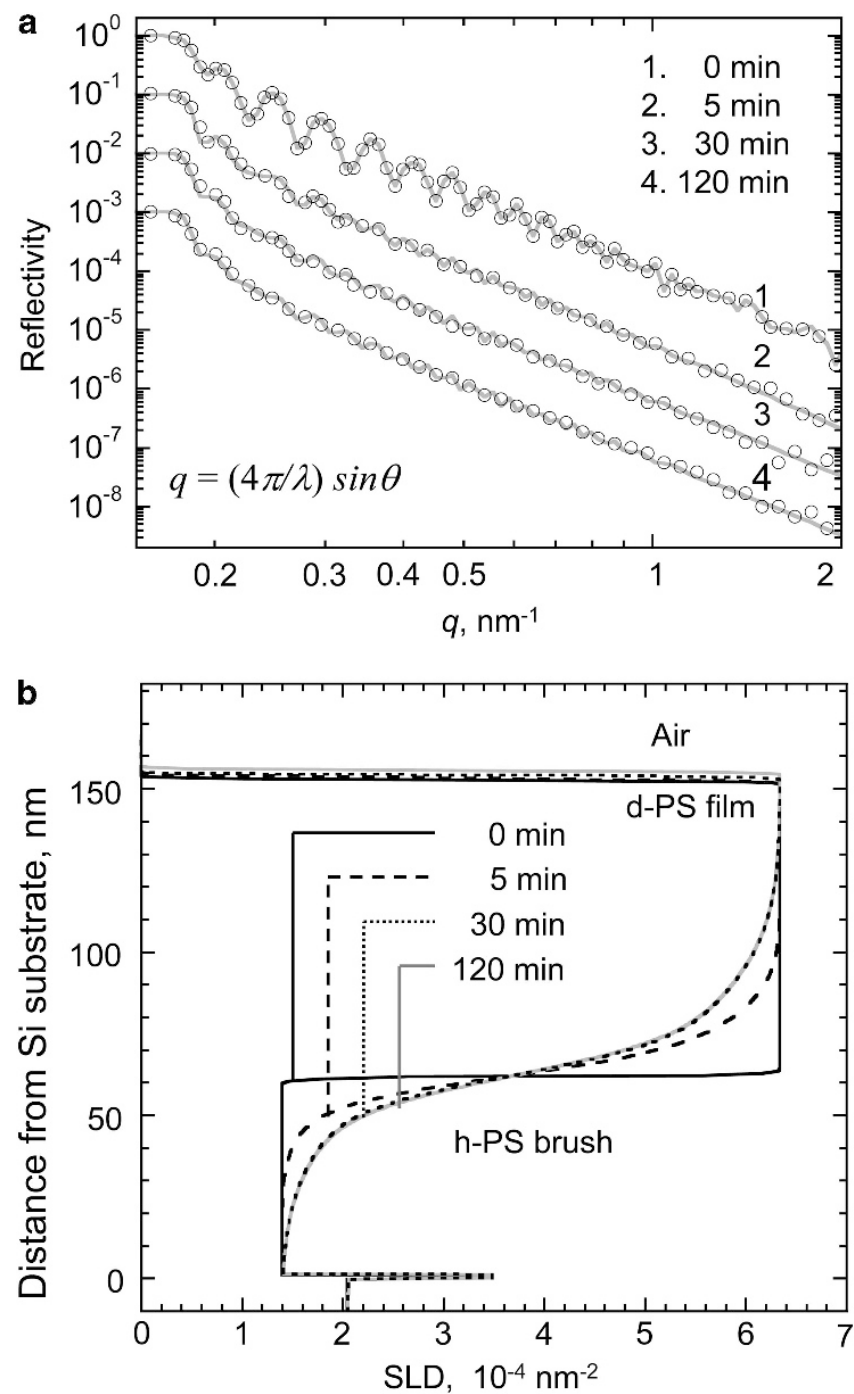

Figure 4 (a) Neutron reflectivity curves (open circles) of hydrogenous polystyrene (hPS)-3 brush/deuterated polystyrene (dPS) bilayer film annealed at $398 \mathrm{~K}$ for $0,5,30$ and $120 \mathrm{~min}$, and the corresponding fit (gray line) calculated on the basis of (b) the scattering length density profiles along with distance from the silicon surface.
Intermixing behavior of broad-MWD hPS brush

To investigate the effects of the MWD on the intermixing behavior, a bilayer consisting of the dPS film and the broad-MWD hPS-3 brush was annealed at $398 \mathrm{~K}$. The dPS/hPS-3 brush bilayer showed intermixing behavior in spite of the dry-brush conditions. As shown in Figure 4, there is a rapid and substantial change in the NR profile after annealing. The fringes in the high $q$ range $\left(0.4-2.0 \mathrm{~nm}^{-1}\right)$ were smeared as the annealing time increased. The SLD profiles in Figure $4 \mathrm{~b}$ show that the sharp interface between the dPS and hPS-3 brush layers becomes much broader after annealing. The intermixing thickness $2 \sigma_{\mathrm{s}}$ estimated by the volume fraction profile in Figure $5 \mathrm{c}$ increased to $10 \mathrm{~nm}$ after annealing for $5 \mathrm{~min}$ and achieved a maximum of $30 \mathrm{~nm}$ after $120 \mathrm{~min}$. The SLD profiles after annealing for 30 and $120 \mathrm{~min}$ are very similar, and the intermixing was almost complete after $30 \mathrm{~min}$. Morphological changes, including the dewetting behavior of the outer dPS layer, were not observed in the atomic force microscopy images for any of the bilayer samples even after annealing for $120 \mathrm{~min}$.

The volume fractions of the hPS-3 brush after annealing in Figure $5 \mathrm{c}$ show characteristic gradient profiles for $-50<z<50 \mathrm{~nm}$
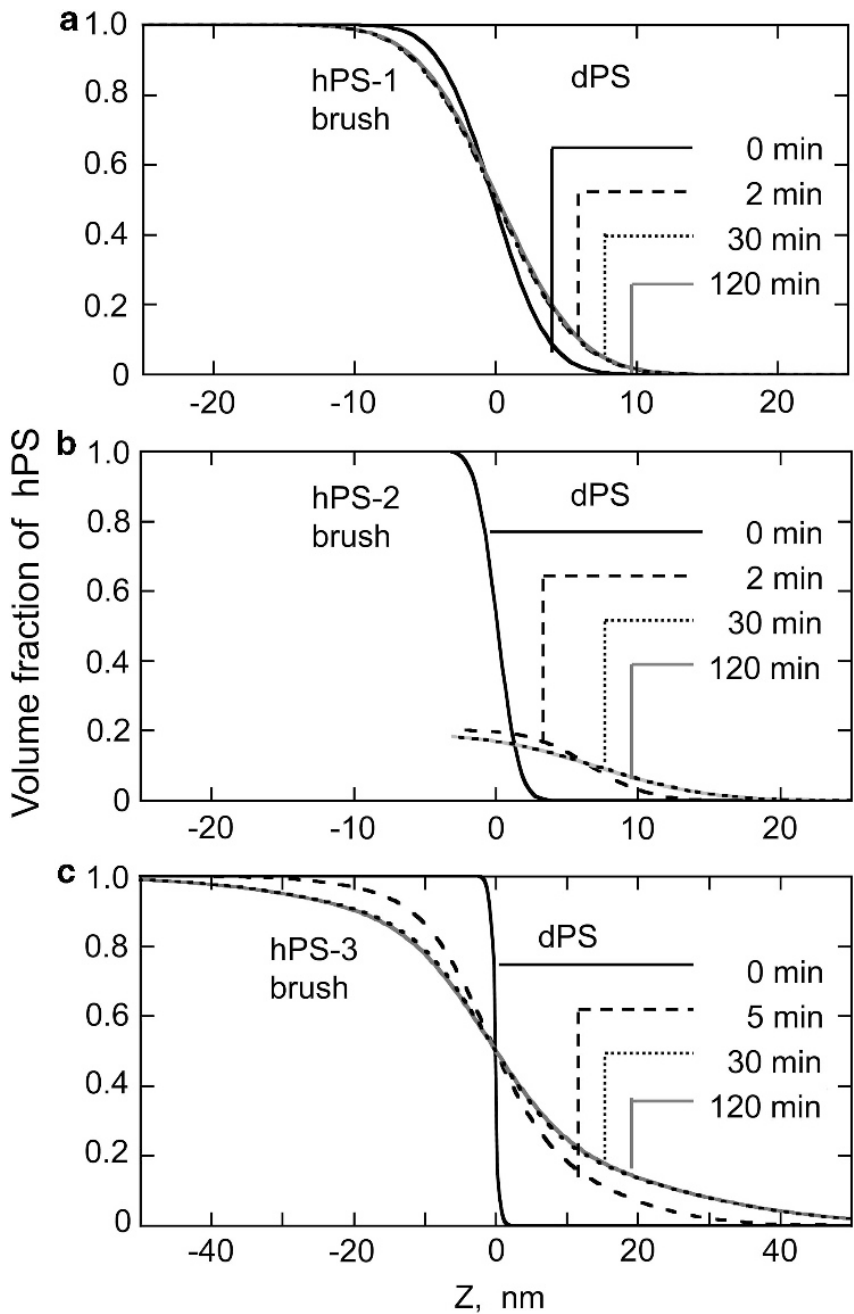

Figure 5 Annealing time dependence of the volume fraction of hPS at the interface between free deuterated polystyrene (dPS) and (a) hydrogenous polystyrene (hPS)-1, (b) hPS-2 and (c) hPS-3 brushes, respectively, at $398 \mathrm{~K}$. 
compared with those of the narrow-MWD hPS-1 brush. The volume fraction distribution curves of the hPS-1 brush at the dPS interface can be approximately described by a parabolic function $\phi_{\mathrm{P}}(x)^{19,20}$ and a Gaussian tail $\phi_{\mathrm{T}}(x):^{21}$

$$
\begin{aligned}
& \phi_{P}(x)=\phi_{0}\left(1-(x / h)^{2}\right)\left(0<x<x_{\mathrm{E}}\right) \\
& \phi_{\mathrm{T}}(x)=M \exp \left(-\beta x^{2}\right)\left(x_{\mathrm{E}}<x\right)
\end{aligned}
$$

where $x, \phi_{0}, h$, and $x_{\mathrm{E}}$ are distance from the substrate surface, the volume fraction at $x=0$, the cutoff thickness when $\phi_{\mathrm{P}}(x)=0$, and the inflection point of the volume fraction curve, respectively; both $M$ and $\beta$ are constants. The $\phi(z)$ profile of the hPS-3 brush, however, shows a long 'tail' extending into the dPS matrix, which cannot be described by this approximation. Although the $\phi(z)$ profile of the hPS-3 brush is better approximated by the two error functions in equations (5) and (6), and by parabolic function and Gaussian tail in equations (8) and (9), the shape of the tail is not well described, as shown in Figure 6. Actually, the parabolic function also gave a good NR fitting curve; however, a slight difference was still observed between the reflectivity curve and the fit (described in Supplementary Information Figures S2 and S3). In addition, the volume fraction forms asymmetric curves at $z<0$ and $z>0$. This type of gradient profile was predicted theoretically by Laub and Koberstein, ${ }^{7}$ who created polymer brushes with various MWDs $\left(M_{\mathrm{w}} / M_{\mathrm{n}}=1.2-3.0\right)$ obeying a logarithmic-normal distribution function and simulated the volume fractions at the interface of the brush and matrix polymer on the basis of a self-consistent field model assuming repulsive, attractive and athermal (neutral) enthalpic brush-matrix interactions. They reported two significant features in the volume fraction profile of brushes with a polydispersity index $>1.5$ : (a) a reduction in the brush volume fraction near the anchoring surface and (b) a long 'foot' (or tail) extending into the matrix phase. The $\phi(z)$ profile of the hPS-3 brush in our experiment is similar to the simulated profile for a brush with $M_{\mathrm{w}} / M_{\mathrm{n}}=2.0$ under athermal conditions. ${ }^{7}$

The long tail of the fraction curve represents the extension of the high molecular weight chains of the hPS into the dPS matrix and the deep penetration of the free dPS chains into the brush layer. These features indicate an increase in the mixing between the brush and matrix. In addition, the entanglement probability was also shown to increase, as the MWD of the brush increased in a simulation study. ${ }^{7}$

(a) $\phi(z)$ of hPS-3

(b) Error function fit

(c) Parabolic function fit

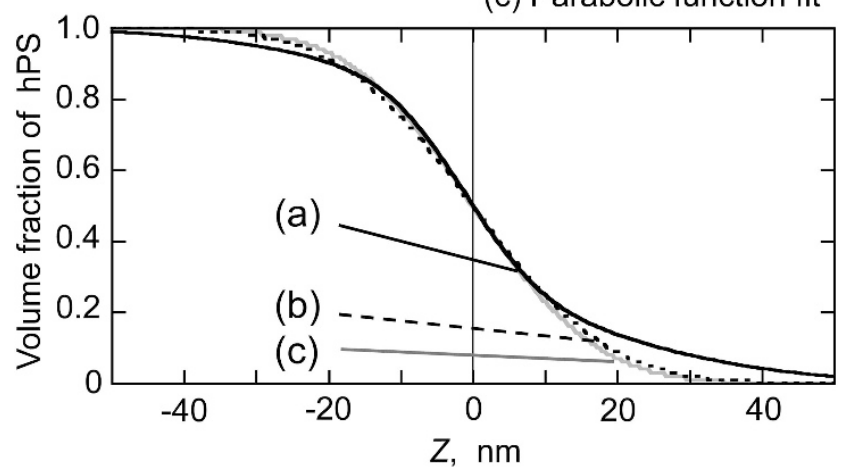

Figure 6 Volume fraction profile of hydrogenous polystyrene (hPS)-3 brush after annealing for $120 \mathrm{~min}$ (a) on the basis of best fit SLD profile, and the corresponding fits by error function (b) and parabolic function connected with Gaussian tail (c).
This is an important issue for the adhesion between the brush on the substrate and the polymer matrix. Brushes at a low grafting density interpenetrate the matrix to form a strong brush-polymer interface; however, the interfacial strength between the substrate and polymer is still weak. A wet brush with a sufficiently high graft density is required to achieve strong substrate-brush and brush-matrix interfaces. A polydisperse brush is likely to be a good candidate for the interfacial adhesion layer.

The contribution of the capillary wave fluctuations at the interface are usually discussed to interpret the results of the NR experiments, but they also have a significant influence on the interface width between the immiscible homopolymer. ${ }^{22,23}$ In addition, the brush fluctuations are much smaller than those of the unbound polymers because one end of the brush chain is tethered to a flat surface. ${ }^{24}$ For this reason, we neglected the fluctuations in these experiments.

Finally, we discuss here the interdiffusion rate of the brush and free polymer chains at the interface. Figure 7 shows a log-log plot of the interfacial widths between the hPS-3 brush and the dPS as a function of the annealing time $t$. The $2 \sigma_{\mathrm{s}}$ value at the hPS brush/dPS bilayer interface quickly increased from 1.6 to $20 \mathrm{~nm}$ within $5 \mathrm{~min}$ after annealing at $398 \mathrm{~K}$ (the $2 \sigma_{\mathrm{s}}$ of the as-prepared sample is not shown in Figure 7). A similarly rapid and significant expansion of the interfacial width was also observed in an 2-min annealing experiment, which was carried out by time-resolved in-situ NR measurements. ${ }^{13}$ The interface width of the sample here slowly increased from 20 to $30 \mathrm{~nm}$ after annealing for $120 \mathrm{~min}$.

In general, the intermixing behavior of two free polymer films consists of two successive time regimes described by Fick- and Rousetype diffusion. ${ }^{10,18,25,26}$ Rouse-type diffusion with a time dependence of $t^{1 / 4}$ is observed for times shorter than the reptation time, and Fickian diffusion has a time dependence of $t^{1 / 2}$ for longer evolution times. ${ }^{25,27,28}$ In contrast to both these diffusion models, the interfacial width of the hPS-3 brush/free dPS shows a time dependence of $2 \sigma_{\mathrm{s}} \sim t^{1 / 8}$ over the $300<t<7200$ interval. We believe that intermixing of the hPS-3 brush and free dPS started immediately after the sample was heated at $398 \mathrm{~K}$ and achieved a metastable state within a few minutes. If we assume Fickian diffusion of the dPS in a short time duration of $0-300 \mathrm{~s}$, the diffusion constant $D$ can be described by

$$
2 \sigma_{\mathrm{s}}=(4 D t)^{1 / 2}
$$

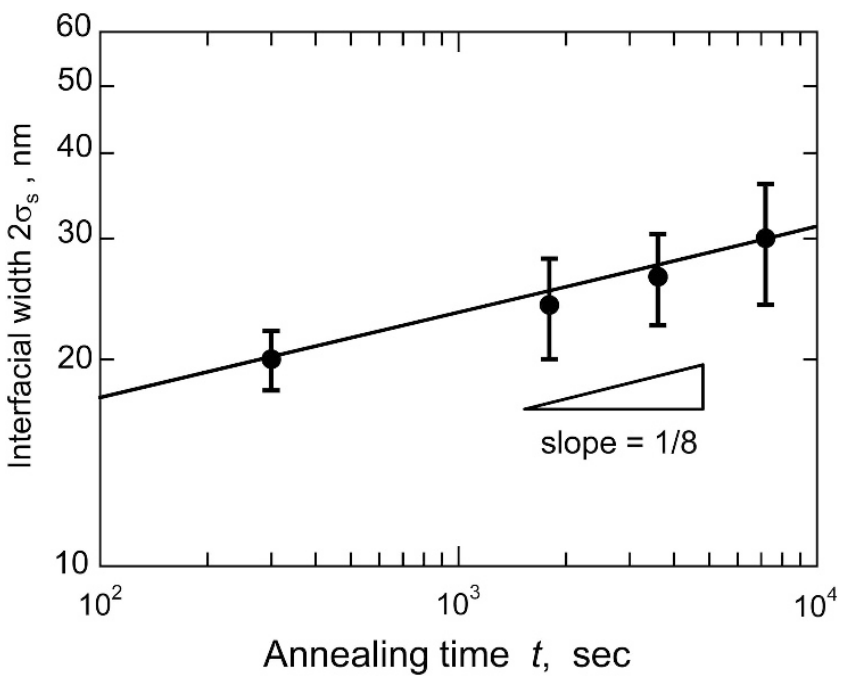

Figure 7 Time evolution of interfacial width of hydrogenous polystyrene (hPS)-3 brush/deuterated polystyrene (dPS) bilyaer film annealed at $398 \mathrm{~K}$. 
which is roughly estimated to be $6.4 \times 10^{-15}\left(\mathrm{~cm}^{2} \mathrm{~s}^{-1}\right)$. An NR experiment for the early stages of annealing was not conducted here, but further work to evaluate the intermixing behavior of the brushes and free polymer is ongoing. NR experiments within such a short annealing time and low annealing temperature are currently being undertaken.

\section{CONCLUSIONS}

We have investigated the effects of the polymer-brush MWD on the intermixing behavior of hPS brushes and free dPS matrices in both the wet and dry states by using an anneal-quench procedure combined with NR measurements. The interfacial width between a bilayer of a high-density hPS brush with a narrow MWD $\left(M_{\mathrm{w}} /\right.$ $\left.M_{\mathrm{n}}=1.12\right)$ and free dPS showed little expansion even after annealing at $398 \mathrm{~K}$ (which is above $T_{\mathrm{g}}$ ), indicating that intermixing did not occur in the dry-brush state as predicted theoretically. However, intermixing of the PS chains proceeded at the interface between the hPS brush with a broad MWD $\left(M_{\mathrm{w}} / M_{\mathrm{n}}=2.02\right)$ and the free dPS by annealing to give a large interface width similar to a wet-brush condition, in spite of the brush having a high grafting density. As the annealing time increased, the interface width increased rapidly from 2 to $20 \mathrm{~nm}$ after $5 \mathrm{~min}$ and then increased slowly to $30 \mathrm{~nm}$ after $120 \mathrm{~min}$. The volume fraction profiles of the hPS brush with the broad MWD showed a characteristic gradient distribution, as the distance from the substrate surface increased. The large interfacial width of a polydisperse brush is expected to improve the adhesive strength between the brush substrate and polymer matrix.

\section{ACKNOWLEDGEMENTS}

The present work was supported by a Grant-in-Aid for the Global COE Program, 'Science for Future Molecular Systems' from the Ministry of Education, Culture, Sports, Science and Technology of Japan. H. A. acknowledges the support of the Japan Science and Technology Agency (JST), ERATO Takahara Soft Interfaces Project.

1 Jones, R. A. L. \& Richards, R. W. Polymers at surface and interfaces 244-292 (Cambridge University Press, New York, 1999).

2 Hasegawa, R., Aoki, Y. \& Doi, M. Optimum graft density for dispersing particles in polymer melts. Macromolecules 29, 6656-6662 (1996).

3 de Gennes, P. G. Conformations of polymers attached to an interface. Macromolecules 13, 1069-1075 (1980).

4 Leibler, L. \& Mourran, A. Mater. Res. Soc. Bull 22, 33 (1997).

5 Ferreira, P. G., Ajdari, A. \& Leibler, L. Scaling law for entropic effects at interfaces between grafted layers and polymer melts. Macromolecules 31, 3994-4003 (1998).

6 Milner, S. T., Witten, T. A. \& Cates, M. E. Effect of polydispersity in the end-grafted polymer brush. Macromolecules 22, 853-861 (1989).
7 Laub, C. F. \& Koberstein, J. T. Effect of brush polydispersity on the interphase between end-grafted brushes and polymeric matrices. Macromolecules 27, 5016-5023 (1994).

8 Queffelec, J., Gaynor, S. G. \& Matyjaszewski, K. Optimization of atom transfer radical polymerization using $\mathrm{Cu}(\mathrm{I}) / \operatorname{Tris}(2$-(dimethylamino)ethyl)amine as a catalyst. Macromolecules 33, 8629 (2000)

9 Yokoyama, H., Kramer, E. J., Hajduk, D. A. \& Bates, F. S. Diffusion in mixtures of asymmetric diblock copolymers with homopolymers. Macromolecules 32, 3353-3359 (1999).

10 Kawaguchi, D., Tanaka, K., Takahara, A. \& Kajiyama, T. Surface mobile layer of polystyrene film below bulk glass transition temperature. Macromolecules 34, 6164-6166 (2001).

11 Mitamura, K., Yamada, N. L., Sagehashi, H., Seto, H., Torikai, N., Sugita, T., Furusaka, M. \& Takahara, A. Advanced neutron reflectometer for investigation on dynamic/static structures of soft-interfaces in J-PARC. J. Phys. Conf. Ser. 272, 012017 (2011).

12 Yamada, N. L., Torikai, N., Mitamura, K., Sagehashi, H., Sato, S., Seto, H., Sugita, T. Goko, S., Furusaka, M., Oda, T., Hino, M., Fujiwara, T., Takahashi, H. \& Takahara, A. Design and performance of horizontal type neutron reflectometer SOFIA at J-PARC/ MLF. Euro. Phys. J. Plus 126, 108 (2011).

13 Mitamura, K., Yamada, N. L., Sagehashi, H., Torikai, N., Arita, H., Terada, M., Kobayashi, M., Sato, S., Seto, H., Goko, S., Furusaka, M., Oda, T., Hino, M., Jinnai, H. \& Takahara, A. Novel neutron reflectometer SOFIA at J-PARC/MLF for in-situ softinterface characterization. Polym. J. 45, 100-108 (2013).

14 Nelson, A. Co-refinement of multiple-constant neutron reflectivity data using MOTOFIT. J. Appl. Crystal. 39, 273-276 (2005).

15 Sears, V. F. Neutron scattering lengths and cross sections. Neutron News 3, 26-37 (1992).

16 Torikai, N. Neutron Reflectometry. in Neutrons in Soft Matter (eds. Imae, T., Kanaya, T., Fususawa, M. \& Torikai, N.) 115-145 (John Wiley \& Sons, Inc., Hoboken, New Jersey, 2011).

17 Konishi, T., Yoshizaki, T., Saito, T., Einaga, Y. \& Yamakawa, H. Mean-square radius of gyration of oligo- and polystyrenes in dilute solutions. Macromolecules 23, 290-297 (1990).

18 Reiter, G. \& Steiner, U. Measurements of polymer diffusion over small distances. A check of reputation arguments. J. Phys. // 1, 659-671 (1991).

19 Milner, S. T., Witten, T. A. \& Cates, M. E. Theory of the grafted polymer brush. Macromolecules 21, 2610-2618 (1988).

20 Migliorini, G. On the corrections to strong-stretching theory for end-confined, charged polymers in a uniform electric field. Macromolecules 43, 9168-9180 (2010).

21 Field, J. B., Toprakcioglu, C., Ball, R. C., Stanley, H. B., Dai, L., Barford, W., Penfold, J., Smith, G. \& Hamilton, W. Determination of end-adsorbed polymer density profiles by neutron reflectivity. Macromolecules 25, 434-439 (1992).

22 Shull, K. R., Mayes, A. M. \& Russell, T. P. Segment distributions in lamellar diblock copolymers. Macromolecules 26, 3929 (1993).

23 Sferrazza, M., Xiao, C., Jones, R. A. L., Bucknall, D. G., Webster, J. \& Penfold, J. Evidence for capillary waves at immiscible polymer/ polymer interfaces. Phys. Rev. Lett. 78, 3693-3696 (1997).

24 Fredrickson, G. H., Ajdari, A., Leibler, L. \& Carton, J. P. Macromolecules 25, 2882 (1992).

25 Karim, A., Mansour, A., Felcher, G. P. \& Russell, T. P. Short-time relaxation at polymeric interfaces. Phys. Rev. B 42, 6846-6850 (1990).

26 Kawaguchi, D., Tanaka, K., Kajiyama, T., Takahara, A. \& Tasaki, S. Mobility gradient in surface region of monodisperse polystyrene films. Macromolecules 36, 1235-1240 (2003).

27 Agrawal, G., Wool, R. P., Dozier, W. D., Felcher, G. P., Russell, T. P. \& Mays, J. W. Short-time interdiffusion at polymer interfaces. Macromolecules 27, 4407-4409 (1994).

28 Welp, K. A., Wool, R. P., Agrawal, G., Satija, S. K., Pispas, S. \& Mays, J. Direct observation of polymer dynamics: mobility comparison between central and end section chain segments. Macromolecules 32, 5127-5138 (1999).

Supplementary information accompanies the paper on Polymer Journal website (http://www.nature.com/pj) 